\title{
Karyotype characterization and nuclear DNA content measurement in Bromeliaceae: State of the art and future perspectives
}

\author{
ANDREI C.P. NUNES and WELLINGTON R. CLARINDO \\ Laboratório de Citogenética, Departamento de Biologia, Centro de Ciências Agrárias, \\ Universidade Federal do Espírito Santo, 29500-000 Alegre, ES, Brasil
}

Manuscript received on May 6, 2014; accepted for publication on July 1, 2014

\begin{abstract}
In Bromeliaceae, cytogenetic and flow cytometry analyses have been performed to clarify systematic and evolutionary aspects. Karyotyping approaches have shown the relatively high chromosome number, similar morphology and small size of the chromosomes. These facts have prevented a correct chromosome counting and characterization. Authors have established a basic chromosome number of $\mathrm{x}=25$ for Bromeliaceae. Recently, one karyomorphological analysis revealed that $x=25$ is no longer the basic chromosome number, whose genome may have a polyploid origin. Besides cytogenetic characterization, the $2 \mathrm{C}$ DNA content of bromeliads has been measured. Nuclear DNA content has varied from $2 \mathrm{C}=0.60$ to $2 \mathrm{C}=3.34$ picograms. Thus, in relation to most angiosperms, the 2C DNA content of Bromeliaceae species as well as their chromosome size can be considered relatively small. In spite of some advances, cytogenetic and flow cytometry data are extremely scarce in this group. In this context, this review reports the state of the art in karyotype characterization and nuclear DNA content measurement in Bromeliaceae, emphasizing the main problems and suggesting prospective solutions and ideas for future research.
\end{abstract}

Key words: allopolyploid, bromeliads, cytogenetics, flow cytometry, genome evolution.

\section{INTRODUCTION}

The Bromeliaceae family belongs to the order Poales (APG III 2009) and comprises about 58 genera and 3,170 species, distributed in tropical and subtropical regions of the American continent (Givnish et al. 2011). Approximately $50 \%$ of the bromeliad species are found in Brazil, occurring in Atlantic Rainforest regions, Caatinga, montane savannas - 'Campos Rupestres', semi-arid regions and tropical savanna - 'Cerrado' (Ceita et al. 2008). For this reason, the Brazil has been considered one of the most important biodiversity Bromeliaceae centers worldwide (Louzada et al. 2010).

Correspondence to: Wellington Ronildo Clarindo

E-mail: wellington.clarindo@ufes.br
Economically, bromeliads have been used for food and fiber production, ornamental purposes and in natural medicine, with the use of the bromelin enzyme. This enzyme is present in the pineapple, Ananas comosus (Linnaeus) Merril, which is also widely explored in agriculture as a commercial fruit. Besides economic aspects, the bromeliads have assumed a substantial role in ecological features. Some species provide concentrated nectar to humming birds and furnish microhabitats for other vegetable species. Thus, the bromeliads have been highlighted as biodiversity enhancers (Versieux 2009, Favoreto et al. 2012).

Cytogenetic studies in Bromeliaceae have been reported, aiming to clarify systematic and 
evolutionary aspects in the group (Favoreto et al. 2012). Initially, cytogenetic researches focused on chromosome counting (Lindschau 1933, Weiss 1965, Marchant 1967). On a second stage, they also reported on the morphology of Bromeliaceae chromosomes (Cotias-de-Oliveira et al. 2000, Palma-Silva et al. 2004, Bellintani et al. 2005, Gitai et al. 2005, Ceita et al. 2008).

Apart from cytogenetic studies, flow cytometry (FCM) analyses have also been performed to measure the nuclear DNA content and base composition (AT\% and $\mathrm{GC} \%$ ) of different Bromeliaceae species, expanding the data about their genome. These analyses have contributed with information for systematic, evolution (Ebert and Till 1997, Ramirez-Morillo and Brown 2001) and genetic diversity studies (Sgorbati et al. 2004, Favoreto et al. 2012).

Despite previous cytogenetic and FCM studies, there is scarce data available for bromeliads, limiting inferences about the evolution of its karyotype. In particular, the most cytogenetic studies have only reported the basic chromosome number, $\mathrm{x}=25$. Considering all this, it is relevant to put together reported cytogenetic and FCM data and the problems being faced, as well as the next steps. Based on this approach, studies on basic chromosome number, ploidy and karyotype evolution in Bromeliaceae could be advanced. Given these aspects, this review was devoted to relating the cytogenetic and FCM data generated so far for this family, showing prospective solutions for old problems and raising new questions.

\section{Cytogenetics of BRomeliaceAe}

At first, cytogenetic studies in Bromeliaceae intended to establish the chromosome number. In 1904, Billings initiated the chromosome analysis of this family, which became more significant after 1933, with studies by Lindschau on 50 species of different Bromeliaceae genera. Subsequently, determination of the chromosome number in some species was accomplished by Marchant (1967), Sharma and
Ghosh (1971), McWilliams (1974), and Varadarajan and Brown (1985). These authors observed a wide diversity of chromosome numbers among species.

Lindschau (1933) proposed that Tillandsioideae has a basic chromosome number of $\mathrm{x}=9$, and Weiss (1965) found $\mathrm{x}=8$; both authors also reported the occurrence of species with different ploidy levels. Marchant (1967) studied 72 Bromeliaceae species, revealing the occurrence of $2 \mathrm{n}=48,50,56,64$, 72, 94, 96, 100 and 126 chromosomes. Based on these results, the author reported that, with the exception of Cryptanthus ( $\mathrm{x}=17)$, Bromeliaceae present a basic chromosome number of $\mathrm{x}=25$. Brown and Gilmartin (1989) suggested that the number $\mathrm{x}=25$ could be derived from hybridization between paleo-diploid species with $\mathrm{x}=8$ and 9, followed by chromosome doubling generating a paleo-tetraploid showing $\mathrm{x}=17$. Subsequently, hybridization between the paleo-tetraploid and the paleo-diploid, with $\mathrm{x}=8$, could have resulted in an allohexaploid exhibiting $\mathrm{x}=8+8+9=25$, then considered the basic chromosome number for the Bromeliaceae family (Figure 1).

On a second stage of cytogenetic studies, besides determining the chromosome number, researchers also characterized the chromosomes of bromeliads morphologically. Several authors reported a chromosome number of $2 n=50$ for most of the species analyzed, with the exception of the genus Cryptanthus, $2 \mathrm{n}=34$, and some polyploid species with $2 \mathrm{n}=100,150$ or 160 . Moreover, morphometric studies revealed the relatively small size of the bromeliad chromosomes (Cotias-deOliveira et al. 2000, 2004, Palma-Silva et al. 2004, Bellintani et al. 2005, Gitai et al. 2005, Ceita et al. 2008).

Initially, Cotias-de-Oliveira et al. (2000) determined the chromosome number of 14 Bromeliaceae species showing $2 \mathrm{n}=50$ and three polyploid species with $2 \mathrm{n}=100$ (Orthophytum burle-marxii LB Smith \& R. W. Read) or $2 \mathrm{n}=150$ (Bromelia laciniosa Martius ex Schultes f. and Orthophytum 


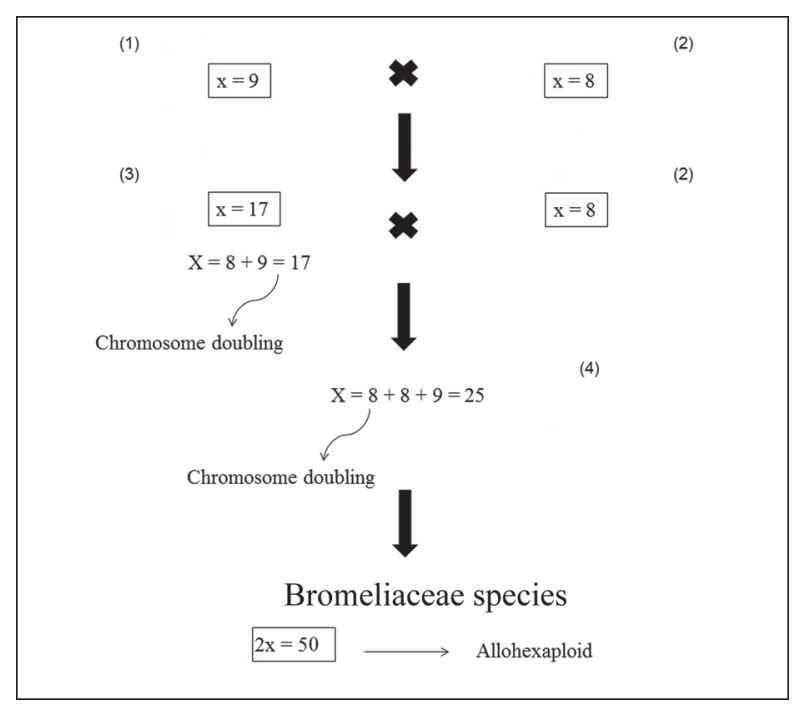

Figure 1 - Schematic representation, which was adapted from Brown and Gilmartin (1989), of the evolutionary process that culminated in the basic number of $\mathrm{x}=25$ chromosomes for the Bromeliaceae family. First, hybridization between paleo-diploid species with $\mathrm{x}=9$ (1) and 8 (2), followed by chromosome doubling generating a paleo-tetraploid with $\mathrm{x}=17$ (3). Subsequently, hybridization between the paleotetraploid (3) and the paleo-diploid with $\mathrm{x}=8$ (2) may have resulted in an allohexaploid with $\mathrm{x}=8+8+9=25$ (4).

maracasense L. B. Smith). These authors also observed that the chromosome size of most species ranged from $0.23 \mu \mathrm{m}$ (chromosome 25) to $1.08 \mu \mathrm{m}$ (chromosome 1). Similarly, Cotias-de-Oliveira et al. (2004) reported a chromosome number of $2 n$ $=50$ for 23 species and $2 \mathrm{n}=100$ for two species, Orthophytum albopictum Philcox and Neoglaziovia variegata (Arruda da Camara) Mez. Moreover, these authors reported a total chromosome size ranging from $0.36 \mu \mathrm{m}$ (chromosome 25) to 1.20 $\mu \mathrm{m}$ (chromosome 1).

Analyzing chromosomal features of Bromeliaceae, Gitai et al. (2005) reported cytological information and chromosome counting of 15 taxa, referring to 19 genera of this family. The basic number $\mathrm{x}=25$ was confirmed, and the occurrence of polyploidy was detected in two species. The species Deinacanthon urbanianum (Nees) Mez had 2n $=160$, while for Bromelia laciniosa Martius ex Schultes f. a number of $2 n=150$ was found. The full size of the chromosomes ranging from $0.50 \mu \mathrm{m}$ (chromosome 25) to $2.72 \mu \mathrm{m}$ (chromosome 1) was observed in different species. Ceita et al. (2008) studied the chromosome number of 18 Bromeliaceae species, finding mostly $2 \mathrm{n}=50$, except for Cryptanthus, with $2 \mathrm{n}$ $=34$. Cryptanthus chromosomes ranged from 0.71 $\mu \mathrm{m}$ (chromosome 17) to $1.25 \mu \mathrm{m}$ (chromosome 1) in length, while other species, showing $2 \mathrm{n}=50$, they ranged from $0.25 \mu \mathrm{m}$ (chromosome 25 ) to $1.5 \mu \mathrm{m}$ (chromosome 1).

As summarized in Table I, $2 \mathrm{n}=50$ prevails in the family. However, cytogenetic studies have shown some variations regarding chromosome number in Cryptanthus genus and some species, as well as discrepancies between the data obtained by analysis of mitotic and meiotic cells. The large number and small size of the chromosomes may have contributed to erroneous counts, which were based on prometaphases or metaphase chromosomes with overlappings, or else due to the possible presence of B chromosomes (Nunes et al. 2013).

The occurrence of relatively small chromosomes has been considered an obstacle for an accurate cytogenetic characterization of most plant species (Carvalho et al. 2008). The chromosome discrimination in Bromeliaceae species has been regarded as particularly laborious, due to their relatively small size and subtle morphological differences (Cotias-de-Oliveira et al. 2000, PalmaSilva et al. 2004, Gitai et al. 2005, Ceita et al. 2008).

Distinct researches have reported chromosome counts in Bromeliaceae based on prophase chromosomes or interphase nuclei, which present blocks of heterochromatin. This kind of chromatin organization, observed before prometaphase, results in chromosomes morphologically elongated and unsuitable for morphometric measurement and karyogram assembly.

As displayed in Figure $2(\mathrm{a}-\mathrm{d})$, different chromatin compaction levels could be observed in cytogenetic analyses. However, interphase 
TABLE I

2C nuclear DNA content, base composition (AT\% and GC\%) and chromosome number (2n) of Bromeliaceae species.

\begin{tabular}{|c|c|c|c|c|c|c|c|}
\hline Genus & Species & $\begin{array}{l}\text { 2C value } \\
\quad(\mathrm{pg})\end{array}$ & $\mathrm{AT} \%$ & $\mathrm{GC} \%$ & $\begin{array}{l}\text { Reference for } 2 \mathrm{C} \\
\text { value and } \mathrm{AT} \% / \mathrm{GC} \%\end{array}$ & $\begin{array}{c}2 \mathrm{n} \\
\text { chromosome } \\
\text { number }\end{array}$ & $\begin{array}{l}\text { Reference for } \\
\text { Chromosome } \\
\text { number }\end{array}$ \\
\hline \multirow{17}{*}{ Aechmea } & $\begin{array}{l}\text { A. nudicaulis var. cuspidata } \\
\text { Baker }\end{array}$ & 0.780 & 64.88 & 35.12 & Favoreto et al. (2012) & - & - \\
\hline & A. ramosa Martius ex Schultes $\mathrm{f}$. & 1.370 & 61.37 & 38.63 & Favoreto et al. (2012) & - & - \\
\hline & A. calyculata (Morren) Baker & - & - & - & - & 50 & $\begin{array}{l}\text { Palma Silva et al. } \\
\text { (2004) }\end{array}$ \\
\hline & A. gamosepala Wittmack & - & - & - & - & 50 & $\begin{array}{l}\text { Palma Silva et al. } \\
\text { (2004) }\end{array}$ \\
\hline & $\begin{array}{l}\text { A. aquilega (Salisbury) } \\
\text { Grisebach }\end{array}$ & - & - & - & - & 50 & $\begin{array}{l}\text { Cotias-de-Oliveira } \\
\text { et al. (2000) }\end{array}$ \\
\hline & $\begin{array}{l}\text { A. blanchetiana (Baker) L. B. } \\
\text { Smith Salvador }\end{array}$ & - & - & - & - & 50 & $\begin{array}{l}\text { Cotias-de-Oliveira } \\
\text { et al. (2000) }\end{array}$ \\
\hline & $\begin{array}{l}\text { A. conifera L. B. Smith Baixa } \\
\text { Gree }\end{array}$ & - & - & - & - & 50 & $\begin{array}{l}\text { Cotias-de-Oliveira } \\
\text { et al. (2000) }\end{array}$ \\
\hline & A. fulgens Brongniart & - & - & - & - & 50 & $\begin{array}{l}\text { Cotias-de-Oliveira } \\
\text { et al. (2000) }\end{array}$ \\
\hline & $\begin{array}{l}\text { A. miniata (Beer) hortus ex } \\
\text { Baker } \\
\text { b var. discolor (Beer) Beer }\end{array}$ & - & - & - & - & 50 & $\begin{array}{l}\text { Cotias-de-Oliveira } \\
\text { et al. (2004) }\end{array}$ \\
\hline & $\begin{array}{l}\text { A. bromeliifolia (Rudge) } \\
\text { Baker }\end{array}$ & - & - & - & - & 50 & Gitaí et al. (2005) \\
\hline & A. caudata Lindman & - & - & - & - & 50 & Ceita et al. (2008) \\
\hline & $\begin{array}{l}\text { A. recurvata (Klotzsch) L. B. } \\
\text { Smith }\end{array}$ & - & - & - & - & 50 & Ceita et al. (2008) \\
\hline & $\begin{array}{l}\text { A. correia-araujoi Pereira \& } \\
\text { Moutinho }\end{array}$ & - & - & - & - & 50 & Ceita et al. (2008) \\
\hline & $\begin{array}{l}\text { A. pineliana (Brongniart ex } \\
\text { Planchon) Baker }\end{array}$ & - & - & - & - & 50 & Ceita et al. (2008) \\
\hline & A. marauensis Leme & - & - & - & - & 50 & Ceita et al. (2008) \\
\hline & A. bicolor L. B. Smith & - & - & - & - & 50 & Ceita et al. (2008) \\
\hline & $\begin{array}{l}\text { A. comata (Gaudichaud) } \\
\text { Baker }\end{array}$ & - & - & - & - & 50 & Ceita et al. (2008) \\
\hline \multirow{2}{*}{ Alcantarea } & A. nahoumi (Leme) R. Grant & - & - & - & - & 50 & Ceita et al. (2008) \\
\hline & A. imperialis (Carrière) Harms & - & - & - & - & 50 & Ceita et al. (2008) \\
\hline \multirow{5}{*}{ Ananas } & A. macrodontes E. Morren & 1.000 & 60.76 & 39.24 & Favoreto et al. (2012) & - & - \\
\hline & $\begin{array}{l}\text { A. bracteatus (Lindley) } \\
\text { Schultes f. }\end{array}$ & 0.920 & - & - & $\begin{array}{l}\text { Arumuganathan and } \\
\text { Earle (1991) }\end{array}$ & - & - \\
\hline & A. comosus Linnaeus Merril & 1.090 & - & - & $\begin{array}{l}\text { Arumuganathan and } \\
\text { Earle (1991) }\end{array}$ & 50 & Gitaí et al. (2005) \\
\hline & A. lucidus Miller & - & - & - & - & 50 & $\begin{array}{l}\text { Cotias-de-Oliveira } \\
\text { et al. }(2000)\end{array}$ \\
\hline & $\begin{array}{l}\text { A. nanus (L. B. Smith) L. B. } \\
\text { Smith }\end{array}$ & - & - & - & - & 50 & $\begin{array}{l}\text { Cotias-de-Oliveira } \\
\text { et al. (2004) }\end{array}$ \\
\hline Ayensua & A. uaipanensis L. B. Smith & 0.860 & - & - & Ebert and Till (1997) & $50 / 100$ & Gitaí et al. (2005) \\
\hline \multirow{4}{*}{ Billbergia } & B. nutans $\mathrm{H}$. Wendle ex Regel & 0.752 & - & - & $\begin{array}{l}\text { Ramírez-Morillo and } \\
\text { Brown (2001) }\end{array}$ & - & - \\
\hline & B. horrida Regel & 0.770 & 64.65 & 35.35 & Favoreto et al. (2012) & - & - \\
\hline & B. euphemiae E. Morren & 0.890 & 62.59 & 37.42 & Favoreto et al. (2012) & 50 & $\begin{array}{l}\text { Cotias-de-Oliveira } \\
\text { et al. (2004) }\end{array}$ \\
\hline & B. tweedieana Baker & 0.950 & 66.05 & 33.95 & Favoreto et al. (2012) & - & - \\
\hline
\end{tabular}


TABLE I (continuation)

\begin{tabular}{|c|c|c|c|c|c|c|c|}
\hline Genus & Species & $\begin{array}{l}2 \mathrm{C} \text { value } \\
\quad(\mathrm{pg})\end{array}$ & $\mathrm{AT} \%$ & $\mathrm{GC} \%$ & $\begin{array}{c}\text { Reference for } 2 \mathrm{C} \\
\text { value and } \mathrm{AT} \% / \mathrm{GC} \%\end{array}$ & $\begin{array}{c}2 \mathrm{n} \\
\text { chromosome } \\
\text { number }\end{array}$ & $\begin{array}{c}\text { Reference for } \\
\text { Chromosome } \\
\text { number }\end{array}$ \\
\hline \multirow{2}{*}{ Billbergia } & $\begin{array}{l}\text { B. chlorosticta Saunders } \\
\text { Hortus }\end{array}$ & - & - & - & - & 50 & $\begin{array}{l}\text { Cotias-de-Oliveira } \\
\text { et al. (2000) }\end{array}$ \\
\hline & B. morelii Brongniart & - & - & - & - & 50 & $\begin{array}{l}\text { Cotias-de-Oliveira } \\
\text { et al. }(2000)\end{array}$ \\
\hline \multirow{2}{*}{ Brocchinia } & B. acuminata L. B. Smith & 0.760 & - & - & Ebert and Till (1997) & - & - \\
\hline & B. tatei L. B. Smith & 0.780 & - & - & Ebert and Till (1997) & - & - \\
\hline \multirow{3}{*}{ Bromelia } & B. antiacantha Bertoloni & 0.810 & 61.57 & 38.43 & Favoreto et al. (2012) & - & - \\
\hline & $\begin{array}{l}\text { B. laciniosa Mart. ex } \\
\text { Schultes }\end{array}$ & - & - & - & 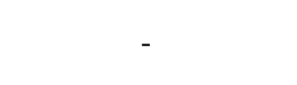 & 50 & $\begin{array}{l}\text { Cotias-de-Oliveira } \\
\text { et al. (2000) }\end{array}$ \\
\hline & $\begin{array}{l}\text { B. plumieri (E. Morren) } \\
\text { L. B. Smith }\end{array}$ & - & - & - & - & 50 & $\begin{array}{l}\text { Cotias-de-Oliveira } \\
\text { et al. }(2000)\end{array}$ \\
\hline \multirow{4}{*}{ Canistropsis } & $\begin{array}{l}\text { C. simulans (Pereira \& } \\
\text { Leme) Leme }\end{array}$ & - & - & - & - & 50 & $\begin{array}{l}\text { Bellintani et al. } \\
\text { (2005) }\end{array}$ \\
\hline & $\begin{array}{l}\text { C. seidelii (L. B. Smith A } \\
\text { Reitz) Leme }\end{array}$ & - & - & - & - & 50 & $\begin{array}{l}\text { Bellintani et al. } \\
\qquad(2005)\end{array}$ \\
\hline & $\begin{array}{l}\text { C. microps ( Pereira \& } \\
\text { Leme) Leme }\end{array}$ & - & - & - & - & 50 & $\begin{array}{l}\text { Bellintani et al. } \\
\qquad(2005)\end{array}$ \\
\hline & C. bilbergioides (Schul.) & 1.000 & 62.64 & 37.36 & Favoreto et al. (2012) & 50 & $\begin{array}{l}\text { Bellintani et al. } \\
(2005)\end{array}$ \\
\hline Canistrum & $\begin{array}{l}\text { C. fosterianum L. B. } \\
\text { Smith }\end{array}$ & - & - & - & - & 50 & $\begin{array}{c}\text { Bellintani et al. } \\
(2005)\end{array}$ \\
\hline Catopsis & C. floribunda L. B. Smith & - & - & - & - & 50 & Gitaí et al. (2005) \\
\hline \multirow{12}{*}{ Cryptanthus } & C. bahianus L. B. Smith & 0.750 & - & - & $\begin{array}{l}\text { Ramírez-Morillo and } \\
\text { Brown (2001) }\end{array}$ & 34 & $\begin{array}{l}\text { Cotias-de-Oliveira } \\
\text { et al. (2000) }\end{array}$ \\
\hline & C. schwackeanus $\mathrm{Mez}$ & 0.710 & - & - & $\begin{array}{l}\text { Ramírez-Morillo and } \\
\text { Brown (2001) }\end{array}$ & 34 & $\begin{array}{l}\text { Ramírez-Morillo } \\
\text { and Brown (2001) }\end{array}$ \\
\hline & C. beuckeri E. Morren & 1.458 & - & - & $\begin{array}{l}\text { Ramírez-Morillo and } \\
\text { Brown (2001) }\end{array}$ & 34 & $\begin{array}{l}\text { Bellintani et al. } \\
\text { (2005) }\end{array}$ \\
\hline & C. acaulis (Lindley) Beer & 1.380 & - & - & $\begin{array}{l}\text { Ramírez-Morillo and } \\
\text { Brown (2001) }\end{array}$ & 34 & $\begin{array}{l}\text { Ramírez-Morillo } \\
\text { and Brown (2001) }\end{array}$ \\
\hline & Cryptanthus sp. & 1.330 & 61.23 & 38.78 & Favoreto et al. (2012) & - & - \\
\hline & C. warren-loosei Leme & - & - & - & - & 34 & Ceita et al. (2008) \\
\hline & C. maritimus L. B. Smith & - & - & - & - & 34 & Ceita et al. (2008) \\
\hline & C. lyman-smith Leme & - & - & - & - & 34 & $\begin{array}{l}\text { Bellintani et al. } \\
\text { (2005) }\end{array}$ \\
\hline & C. vexatus Leme & - & - & - & - & 34 & $\begin{array}{l}\text { Bellintani et al. } \\
\qquad(2005)\end{array}$ \\
\hline & $\begin{array}{l}\text { C. bromelioides Otto \& } \\
\text { F. Dietr. }\end{array}$ & - & - & - & - & 34 & $\begin{array}{l}\text { Sharma and } \\
\text { Ghosh (1971) }\end{array}$ \\
\hline & $\begin{array}{l}\text { C. praetextus E. Morren } \\
\text { ex Baker }\end{array}$ & - & - & - & - & 34 & $\begin{array}{l}\text { Sharma and } \\
\text { Ghosh (1971) }\end{array}$ \\
\hline & C. zonatus (Visiani) Beer & - & - & - & - & 36 & Lindschau (1933) \\
\hline Deinacanthon & $\begin{array}{l}\text { D. urbanianum }(\mathrm{Mez}) \\
\text { Mez }\end{array}$ & - & - & - & - & 50 & Gitaí et al. (2005) \\
\hline \multirow{3}{*}{ Deuterocohnia } & $\begin{array}{l}\text { D. longipetala (Baker) } \\
\mathrm{Mez}\end{array}$ & 0.740 & - & - & Ebert and Till (1997) & - & - \\
\hline & D. schreiteri Castellanos & 0.800 & - & - & Ebert and Till (1997) & - & - \\
\hline & $\begin{array}{l}\text { D. lorentziana }(\mathrm{Mez}) \\
\text { Spencer \& L. B. Smith }\end{array}$ & - & - & - & - & 50 & Gitaí et al. (2005) \\
\hline Dyckia & D. estevesii Rauh & 1.600 & - & - & Ebert and Till (1997) & - & - \\
\hline
\end{tabular}


TABLE I (continuation)

\begin{tabular}{|c|c|c|c|c|c|c|c|}
\hline Genus & Species & $\begin{array}{c}2 \mathrm{C} \\
\text { value } \\
(\mathrm{pg})\end{array}$ & $\mathrm{AT} \%$ & $\mathrm{GC} \%$ & $\begin{array}{c}\text { Reference for } 2 \mathrm{C} \\
\text { value and } \mathrm{AT} \% / \\
\mathrm{GC} \%\end{array}$ & $\begin{array}{c}2 \mathrm{n} \\
\text { chromosome } \\
\text { number }\end{array}$ & $\begin{array}{l}\text { Reference for } \\
\text { Chromosome } \\
\text { number }\end{array}$ \\
\hline \multirow{3}{*}{ Dyckia } & D. floribunda Grisebach & 1.580 & - & - & Ebert and Till (1997) & - & - \\
\hline & D. platyphylla L. B. Smith & - & - & - & - & 50 & $\begin{array}{l}\text { Cotias-de-Oliveira } \\
\text { et al. (2004) }\end{array}$ \\
\hline & D. saxatilis $\mathrm{Mez}$ & - & - & - & - & 50 & Gitaí et al. (2005) \\
\hline \multirow{3}{*}{ Encholirium } & E. irwinii L. B. Smith & 1.740 & - & - & Ebert and Till (1997) & - & - \\
\hline & Encholirium sp. & 1.740 & - & - & Ebert and Till (1997) & - & - \\
\hline & E. spectabile Mart. ex Schult.f. & - & - & - & - & 50 & $\begin{array}{l}\text { Cotias-de-Oliveira } \\
\text { et al. (2000) }\end{array}$ \\
\hline \multirow{2}{*}{ Fascicularia } & $\begin{array}{l}\text { F. bicolor (Ruiz \& Pavon) Mez } \\
\text { ssp. bicolor E.C. Nelson \& } \\
\text { Zizka }\end{array}$ & - & - & - & - & 50 & Gitaí et al. (2005) \\
\hline & $\begin{array}{l}\text { F. bicolor (Ruiz \& Pavon) Mez } \\
\text { ssp. canaliculata E.C. Nelson } \\
\text { \& Zizka }\end{array}$ & - & - & - & - & 50 & Gitaí et al. (2005) \\
\hline \multirow{2}{*}{ Fosterella } & $\begin{array}{l}\text { F. penduliflora (C.H. Wright) } \\
\text { L.B. Smith }\end{array}$ & 1.860 & - & - & Ebert and Till (1997) & - & - \\
\hline & $\begin{array}{l}\text { F. villosula (Harms) L. B. } \\
\text { Smith }\end{array}$ & 1.860 & - & - & Ebert and Till (1997) & - & - \\
\hline \multirow{2}{*}{ Greigia } & $\begin{array}{l}\text { G. spec. nov. (aff. G. mulfordii } \\
\text { var. micrantha) }\end{array}$ & - & - & - & - & 50 & Gitaí et al. (2005) \\
\hline & $\begin{array}{l}\text { G. sphacelata (Ruiz \& Pavon) } \\
\text { Regel }\end{array}$ & - & - & - & - & 50 & Gitaí et al. (2005) \\
\hline \multirow{2}{*}{ Hechtia } & H. epigyna Harms & 0.960 & - & - & Ebert and Till (1997) & - & - \\
\hline & H. matudae L. B. Smith & 0.940 & - & - & Ebert and Till (1997) & - & - \\
\hline \multirow{7}{*}{ Hohenbergia } & H. catingae Ule var. catingae & - & - & - & - & 50 & $\begin{array}{l}\text { Cotias-de-Oliveira } \\
\text { et al. (2000) }\end{array}$ \\
\hline & H. littoralis L. B. Smith & - & - & - & - & 50 & $\begin{array}{l}\text { Cotias-de-Oliveira } \\
\text { et al. (2000) } \\
\text { Cotias-de-Oliveira } \\
\text { et al. (2000) }\end{array}$ \\
\hline & $\begin{array}{l}\text { H. stellata Schultes Baixa Gree } \\
\text { H. blanchetti (Baker) E. } \\
\text { Morren ex Mez }\end{array}$ & - & - & - & - & $\begin{array}{l}50 \\
50\end{array}$ & Ceita et al. (2008) \\
\hline & H. pennae Pereira & - & - & - & - & 50 & $\begin{array}{l}\text { Bellintani et al. } \\
\qquad(2005)\end{array}$ \\
\hline & $\begin{array}{l}\text { H. castelanosii L. B. Smith a } \\
\text { Read }\end{array}$ & - & - & - & - & 50 & $\begin{array}{l}\text { Bellintani et al. } \\
\qquad(2005)\end{array}$ \\
\hline & $\begin{array}{l}\text { H. correia-araujoi Pereira \& } \\
\text { Moutinho }\end{array}$ & - & - & - & - & 50 & $\begin{array}{l}\text { Pereira \& } \\
\text { Moutinho }\end{array}$ \\
\hline & H. aff. utriculosa Ule & - & - & - & - & 50 & $\begin{array}{l}\text { Cotias-de-Oliveira } \\
\text { et al. (2000) }\end{array}$ \\
\hline Navia & N. splendens L. B. Smith & 1.420 & - & - & Ebert and Till (1997) & - & - \\
\hline Neoglaziovia & $\begin{array}{l}\text { N. variegata } \\
\text { (Arruda de Camara) Mez }\end{array}$ & - & - & - & - & 100 & $\begin{array}{l}\text { Cotias-de-Oliveira } \\
\text { et al. (2000) }\end{array}$ \\
\hline \multirow{4}{*}{ Neoregelia } & N. aff. Simulans L. B. Smith & 0.980 & 62.76 & 37.24 & $\begin{array}{l}\text { Favoreto et al. } \\
\quad(2012)\end{array}$ & - & 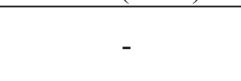 \\
\hline & $\begin{array}{l}\text { N. carcharodon (Baker) L. B. } \\
\text { Smith }\end{array}$ & - & - & - & - & 50 & $\begin{array}{l}\text { Cotias-de-Oliveira } \\
\text { et al. (2004) }\end{array}$ \\
\hline & N.hoehneana L. B. Smith & - & - & - & - & 50 & $\begin{array}{l}\text { Cotias-de-Oliveira } \\
\text { et al. (2004) }\end{array}$ \\
\hline & N. laevis (Mez) L. B. Smith & - & - & - & - & 50 & $\begin{array}{l}\text { Cotias-de-Oliveira } \\
\text { et al. (2004) }\end{array}$ \\
\hline
\end{tabular}


TABLE I (continuation)

\begin{tabular}{|c|c|c|c|c|c|c|c|}
\hline Genus & Species & $\begin{array}{c}2 \mathrm{C} \\
\text { value } \\
(\mathrm{pg}) \\
\end{array}$ & $\mathrm{AT} \%$ & $\mathrm{GC} \%$ & $\begin{array}{c}\text { Reference for } 2 \mathrm{C} \\
\text { value and } \mathrm{AT} \% / \mathrm{GC} \%\end{array}$ & $\begin{array}{c}2 \mathrm{n} \\
\text { chromosome } \\
\text { number }\end{array}$ & $\begin{array}{c}\text { Reference for } \\
\text { Chromosome } \\
\text { number }\end{array}$ \\
\hline \multirow{11}{*}{ Neoregelia } & $\begin{array}{l}\text { N. johannis (Carrière) L. B. } \\
\text { Smith }\end{array}$ & - & - & - & - & 50 & $\begin{array}{c}\text { Cotias-de-Oliveira et } \\
\text { al. (2004) }\end{array}$ \\
\hline & N. wilsoniana M.B. Foster & - & - & - & - & 50 & $\begin{array}{l}\text { Cotias-de-Oliveira et } \\
\text { al. (2004) }\end{array}$ \\
\hline & N. rutilans E. Morren & - & - & - & - & 50 & $\begin{array}{l}\text { Cotias-de-Oliveira et } \\
\text { al. (2004) }\end{array}$ \\
\hline & N. longiflorum Ule & - & - & - & - & 50 & $\begin{array}{c}\text { Cotias-de-Oliveira et } \\
\text { al. }(2004)\end{array}$ \\
\hline & N. procerum Lindman & - & - & - & - & 50 & Ceita et al. (2008) \\
\hline & $N$. tenebrosa Leme & - & - & - & - & 50 & Ceita et al. (2008) \\
\hline & $\begin{array}{l}\text { N. leucophoea (Baker) L. } \\
\text { B. Smith }\end{array}$ & - & - & - & - & 50 & $\begin{array}{l}\text { Bellintani } \\
\text { et al. }(2005)\end{array}$ \\
\hline & $\begin{array}{l}\text { N. longisepala Pereira \& } \\
\text { Penna }\end{array}$ & - & - & - & - & 50 & Pereira \& Penna \\
\hline & $\begin{array}{l}\text { N. cruenta (R. Graham) L. } \\
\text { B. Smith }\end{array}$ & - & - & - & - & 50 & $\begin{array}{l}\text { Bellintani } \\
\text { et al. (2005) }\end{array}$ \\
\hline & $\begin{array}{l}\text { N. carolinae (Beer) L. B. } \\
\text { Smith }\end{array}$ & - & - & - & - & 50 & $\begin{array}{l}\text { Bellintani } \\
\text { et al. }(2005)\end{array}$ \\
\hline & $\begin{array}{l}\text { N. bahiana (Ule) L. B. } \\
\text { Smith }\end{array}$ & - & - & - & - & 50 & $\begin{array}{c}\text { Bellintani } \\
\text { et al. }(2005)\end{array}$ \\
\hline \multirow{4}{*}{ Nidularium } & N. lyman-smithii Leme & - & - & - & - & 50 & Ceita et al. (2008) \\
\hline & N. scheremetiewii Regel & - & - & - & - & 50 & Ceita et al. (2008) \\
\hline & $\begin{array}{l}N . \text { innocentii var. } \\
\text { innocentii Lemaire }\end{array}$ & - & - & - & - & 50 & Ceita et al. (2008) \\
\hline & $\begin{array}{l}\text { N. innocentii x Neoregelia } \\
\text { johannis }\end{array}$ & - & - & - & - & 50 & Ceita et al. (2008) \\
\hline \multirow[b]{2}{*}{ Ochagavia } & O. elegans R. Philippi & - & - & - & - & 50 & Gitaí et al. (2005) \\
\hline & $\begin{array}{l}\text { O.litoralis (Phil.) Zizka, } \\
\text { Trumpler \& Zoellner }\end{array}$ & - & - & - & - & 50 & Gitaí et al. (2005) \\
\hline \multirow{11}{*}{ Orthophytum } & $\begin{array}{l}\text { O. saxicola (Ule) L. B. } \\
\text { Smith }\end{array}$ & 0.640 & - & - & $\begin{array}{l}\text { Ramírez-Morillo and } \\
\text { Brown (2001) }\end{array}$ & 50 & $\begin{array}{l}\text { Ramírez-Morillo and } \\
\text { Brown (2001) }\end{array}$ \\
\hline & $\begin{array}{l}\text { O. maracasense L. B. } \\
\text { Smith }\end{array}$ & - & - & - & 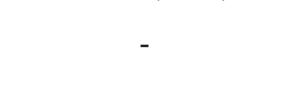 & 150 & $\begin{array}{l}\text { Cotias-de-Oliveira et } \\
\text { al. (2000) }\end{array}$ \\
\hline & $\begin{array}{l}\text { O. burle-marxii L. B. } \\
\text { Smith \& Rangel }\end{array}$ & - & - & - & - & 100 & $\begin{array}{c}\text { Cotias-de-Oliveira et } \\
\text { al. }(2000)\end{array}$ \\
\hline & O. albopictum Philcox & - & - & - & - & 100 & Louzada et al. (2010) \\
\hline & $\begin{array}{l}\text { O. amoenum (Ule) L. B. } \\
\text { Smith }\end{array}$ & - & - & - & - & 100 & Louzada et al. (2010) \\
\hline & O. disjunctum L. B. Smith & - & - & - & - & 50 & Louzada et al. (2010) \\
\hline & O. hatschbachii Leme & - & - & - & - & 50 & Louzada et al. (2010) \\
\hline & $\begin{array}{l}\text { O. mucugense Wand.\& A. } \\
\text { A. Conc. }\end{array}$ & - & - & - & - & 50 & Louzada et al. (2010) \\
\hline & $\begin{array}{l}\text { O. supthutii E. Gross \& } \\
\text { Barthlott }\end{array}$ & - & - & - & - & 50 & Louzada et al. (2010) \\
\hline & $\begin{array}{l}\text { O. ophiuroides Louzada } \\
\text { \& We }\end{array}$ & - & - & - & - & 50 & Louzada et al. (2010) \\
\hline & O. vagans M. B. Foster & - & - & - & - & 50 & Louzada et al. (2010) \\
\hline \multirow{2}{*}{ Pitcairnia } & $\begin{array}{l}\text { P.flammea (L. B. Smith) L. } \\
\text { B. Smith }\end{array}$ & 1.44 & 64.28 & 35.72 & Nunes et al. (2013) & 50 & Nunes et al. (2013) \\
\hline & $\begin{array}{l}\text { P. feliciana (A. Chevalier) } \\
\text { Harms \& Mildbraed }\end{array}$ & 0.600 & - & - & Ebert and Till (1997) & - & - \\
\hline
\end{tabular}


TABLE I (continuation)

\begin{tabular}{|c|c|c|c|c|c|c|c|}
\hline Genus & Species & $\begin{array}{c}2 \mathrm{C} \\
\text { value } \\
(\mathrm{pg}) \\
\end{array}$ & $\mathrm{AT} \%$ & $\mathrm{GC} \%$ & $\begin{array}{c}\text { Reference for } 2 \mathrm{C} \\
\text { value and } \mathrm{AT} \% / \mathrm{GC} \%\end{array}$ & $\begin{array}{c}2 \mathrm{n} \\
\text { chromosome } \\
\text { number }\end{array}$ & $\begin{array}{c}\text { Reference for } \\
\text { Chromosome } \\
\text { number }\end{array}$ \\
\hline \multirow{28}{*}{ Pitcairnia } & P. heterophylla (Lindley) Beer & 0.880 & - & - & Ebert and Till (1997) & - & - \\
\hline & P. prolifera Rauh & 0.840 & - & - & Ebert and Till (1997) & - & - \\
\hline & P. cardenasii L. B. Smith & 1.020 & - & - & Ebert and Till (1997) & - & - \\
\hline & P. rectiflora Rauh & 1.200 & - & - & Ebert and Till (1997) & - & - \\
\hline & P. sceptrigera Mez & 1.200 & - & - & Ebert and Till (1997) & - & - \\
\hline & P. piepenbringii Rauh \& E. Gross & 1.200 & - & - & Ebert and Till (1997) & - & - \\
\hline & P. poeppigiana $\mathrm{Mez}$ & 1.200 & - & - & Ebert and Till (1997) & - & - \\
\hline & P. atrorubens (Beer) Baker & 1.200 & - & - & Ebert and Till (1997) & 50 & Gitaí et al. (2005) \\
\hline & P. chiapensis Mirea & 1.220 & - & - & Ebert and Till (1997) & - & -- \\
\hline & P. spicata (Lamarck) Mez & 1.220 & - & - & Ebert and Till (1997) & - & - \\
\hline & P. aphelandriflora L. B. Smith & 1.240 & - & - & Ebert and Till (1997) & - & - \\
\hline & P. pomacochae Rauh & 1.240 & - & - & Ebert and Till (1997) & - & - \\
\hline & P. aureobrunnea Rauh & 1.120 & - & - & Ebert and Till (1997) & - & - \\
\hline & P. yaupi-bajaensis Rauh & 1.120 & - & - & Ebert and Till (1997) & - & - \\
\hline & P. riparia $\mathrm{Mez}$ & 1.140 & - & - & Ebert and Till (1997) & - & - \\
\hline & P. halophila L. B. Smith & 1.080 & - & - & Ebert and Till (1997) & - & - \\
\hline & P. micotrinensis R.W. Read & 1.100 & - & - & Ebert and Till (1997) & - & - \\
\hline & P. tabuliformis Linden & 1.100 & - & - & Ebert and Till (1997) & - & - \\
\hline & P. angustifolia Soleer in Aiton & 1.060 & - & - & Ebert and Till (1997) & - & - \\
\hline & P. albomarginata L. B. Smith & 1.280 & - & - & Ebert and Till (1997) & - & - \\
\hline & P. heerdeae E. Gross \& Rauh & 1.180 & - & - & Ebert and Till (1997) & - & - \\
\hline & P. palmoides Mez \& Sodiro & 1.180 & - & - & Ebert and Till (1997) & - & - \\
\hline & P. paraguayensis L. B. Smith & 1.360 & - & - & Ebert and Till (1997) & - & - \\
\hline & $\begin{array}{l}\text { P. venezuelana L.B. Smith \& } \\
\text { Steyermark }\end{array}$ & 1.360 & - & - & Ebert and Till (1997) & - & - \\
\hline & P. villetaensis Rauh & 1.260 & - & - & Ebert and Till (1997) & - & - \\
\hline & P. andrena Linden & 1.300 & - & - & Ebert and Till (1997) & - & - \\
\hline & P. schultzei Harms & 1.360 & - & - & Ebert and Till (1997) & - & - \\
\hline & P.grafii Rauh & 1.340 & - & - & Ebert and Till (1997) & - & - \\
\hline \multirow{2}{*}{ Portea } & P. silveirae $\mathrm{Mez}$ & - & - & - & - & 50 & $\begin{array}{l}\text { Cotias-de-Oliveira } \\
\text { et al. (2004) }\end{array}$ \\
\hline & P. greiflora Philcox & - & - & - & - & 50 & $\begin{array}{l}\text { Cotias-de-Oliveira } \\
\text { et al. (2004) }\end{array}$ \\
\hline \multirow{3}{*}{ Puya } & P. mirabilis (Mez) L. B. Smith & 0.880 & - & - & Ebert and Till (1997) & 50 & Gitaí et al. (2005) \\
\hline & P. stenothyrsa (Baker) Mez & 0.940 & - & - & Ebert and Till (1997) & - & - \\
\hline & P. raimondii Harms & 1.130 & - & - & Sgorbati et al. (2004) & - & - \\
\hline \multirow{2}{*}{ Quesnelia } & Q. arvenis (Vellozo) Mez & - & - & - & er & 50 & $\begin{array}{l}\text { Cotias-de-Oliveira } \\
\text { et al. (2004) }\end{array}$ \\
\hline & $\begin{array}{l}\text { Q. edmundoi L. B. Smith b var. } \\
\text { rubrobracteata E. Pereira }\end{array}$ & - & - & - & - & 50 & $\begin{array}{l}\text { Cotias-de-Oliveira } \\
\text { et al. (2004) }\end{array}$ \\
\hline \multirow{5}{*}{ Vriesea } & V. racinae L. B. Smith & 1.190 & 60.26 & 39.74 & Favoreto et al. (2012) & - & - \\
\hline & V. scalaris E. Morren & 1.110 & 60.77 & 39.23 & Favoreto et al. (2012) & - & - \\
\hline & V. carinata Wawra & - & - & - & 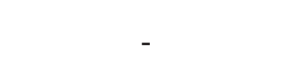 & 50 & $\begin{array}{l}\text { Palma Silva } \\
\text { et al. (2004) }\end{array}$ \\
\hline & $\begin{array}{l}\text { V. erytrodactylon (E. Morren) E. } \\
\text { Morren ex. Mez }\end{array}$ & - & - & - & - & 50 & $\begin{array}{l}\text { Palma Silva } \\
\text { et al. (2004) }\end{array}$ \\
\hline & V. flammea L. B. Smith & - & - & - & - & 50 & $\begin{array}{l}\text { Palma Silva } \\
\text { et al. (2004) }\end{array}$ \\
\hline
\end{tabular}


TABLE I (continuation)

\begin{tabular}{|c|c|c|c|c|c|c|c|}
\hline Genus & Species & $\begin{array}{l}2 \mathrm{C} \text { value } \\
\quad(\mathrm{pg})\end{array}$ & $\mathrm{AT} \%$ & $\mathrm{GC} \%$ & $\begin{array}{l}\text { Reference for } 2 \mathrm{C} \text { value } \\
\text { and } \mathrm{AT} \% / \mathrm{GC} \%\end{array}$ & $\begin{array}{l}\text { 2n chromosome } \\
\text { number }\end{array}$ & $\begin{array}{c}\text { Reference for } \\
\text { Chromosome } \\
\text { number }\end{array}$ \\
\hline \multirow{10}{*}{ Vriesea } & V. friburgensis $\mathrm{Mez}$ & - & - & - & - & 50 & $\begin{array}{l}\text { Palma Silva } \\
\text { et al. (2004) }\end{array}$ \\
\hline & $\begin{array}{l}V . \text { guttata Linden } \\
\text { e Ere }\end{array}$ & - & - & - & - & 50 & $\begin{array}{l}\text { Palma Silva } \\
\text { et al. (2004) }\end{array}$ \\
\hline & $\begin{array}{l}\text { V. incurvata } \\
\text { Gaudchaud }\end{array}$ & - & - & - & - & 50 & $\begin{array}{l}\text { Palma Silva } \\
\text { et al. (2004) }\end{array}$ \\
\hline & $\begin{array}{l}\text { V. platynema } \\
\text { Gaudchaud }\end{array}$ & - & - & - & - & 50 & $\begin{array}{l}\text { Palma Silva } \\
\text { et al. (2004) }\end{array}$ \\
\hline & $\begin{array}{l}\text { V. platzmannii E. } \\
\text { Morren }\end{array}$ & - & - & - & - & 50 & $\begin{array}{l}\text { Palma Silva } \\
\text { et al. (2004) }\end{array}$ \\
\hline & $\begin{array}{l}\text { V. psittacina } \\
\text { (Hooker) Lindley }\end{array}$ & - & - & - & - & 50 & $\begin{array}{l}\text { Palma Silva } \\
\text { et al. (2004) }\end{array}$ \\
\hline & $\begin{array}{l}V . \text { fosteriana L. B. } \\
\text { Smith }\end{array}$ & - & - & - & - & 50 & $\begin{array}{l}\text { Cotias-de-Oliveira } \\
\text { et al. (2004) }\end{array}$ \\
\hline & $\begin{array}{l}V . \text { picta }(\text { Mez et } \\
\text { Wercklé) L. B. } \\
\text { Smith et Pittendr. }\end{array}$ & - & - & - & - & 50 & $\begin{array}{l}\text { Cotias-de-Oliveira } \\
\text { et al. (2004) }\end{array}$ \\
\hline & V. botafogensis $\mathrm{Mez}$ & - & - & - & - & 50 & $\begin{array}{l}\text { Cotias-de-Oliveira } \\
\text { et al. (2004) }\end{array}$ \\
\hline & $\begin{array}{l}\text { V. saundersii } \\
\text { (Carrière) E. Morren } \\
\text { ex Mez }\end{array}$ & - & - & - & - & 50 & $\begin{array}{l}\text { Cotias-de-Oliveira } \\
\text { et al. (2004) }\end{array}$ \\
\hline \multirow{5}{*}{ Tillandsia } & T. stricta Soleer & 1.200 & 61.94 & 38.06 & Favoreto et al. (2012) & - & - \\
\hline & $\begin{array}{l}\text { T. cyanea Linden ex } \\
\text { K. Koch }\end{array}$ & 2.200 & - & - & Zonneveld et al. (2005) & - & - \\
\hline & $\begin{array}{l}\text { T. usneoides } \\
\text { (Linnaeus) Linnaeus }\end{array}$ & 2.500 & - & - & Zonneveld et al. (2005) & - & - \\
\hline & $\begin{array}{l}\text { T. loliacea Martius } \\
\text { ex Schultes f. }\end{array}$ & 3.340 & 60.75 & 39.25 & Favoreto et al. (2012) & - & - \\
\hline & $\begin{array}{l}\text { T. dodsonii L. B. } \\
\text { Smith }\end{array}$ & - & - & - & - & 50 & Ceita et al. (2008) \\
\hline \multirow{2}{*}{ Wittrockia } & $\begin{array}{l}\text { W. gigantea (Baker) } \\
\text { Leme }\end{array}$ & - & - & - & - & 160 & Gitaí et al. (2005) \\
\hline & $\begin{array}{l}\text { W. spiralipetala } \\
\text { Leme }\end{array}$ & - & - & - & - & 50 & $\begin{array}{l}\text { Bellintani } \\
\text { et al. (2005) }\end{array}$ \\
\hline
\end{tabular}

and prophase chromosomes (Figure 2a, c), which present low chromatin compaction level, are inadequate for morphometric measurements, as they prevent correct karyomorphological analyses. As a result thereof, erroneous chromosome counts and characterization are made, generating incorrect data from the karyotype study.

Recently, Nunes et al. (2013) established a protocol for obtaining metaphase chromosomes of Pitcairnia flammea (L. B. Smith) L. B. Smith. The authors used the technique of cell dissociation of enzymatically macerated roots and subsequent air drying of the slides. These procedures provided chromosomes with well-defined primary constrictions, few overlaps, deformations or cytoplasmic chromatin fragments (Figure 2d). Based on these results, the authors were able to perform morphometric analyses, pairing of homologous chromosomes and assembly of a Bromeliaceae karyogram. Analyzing the karyogram of P. Alammea, they detected the presence of grouped pairs of cytogenetically identical chromosomes. Thus, the authors inferred that the basic number for 


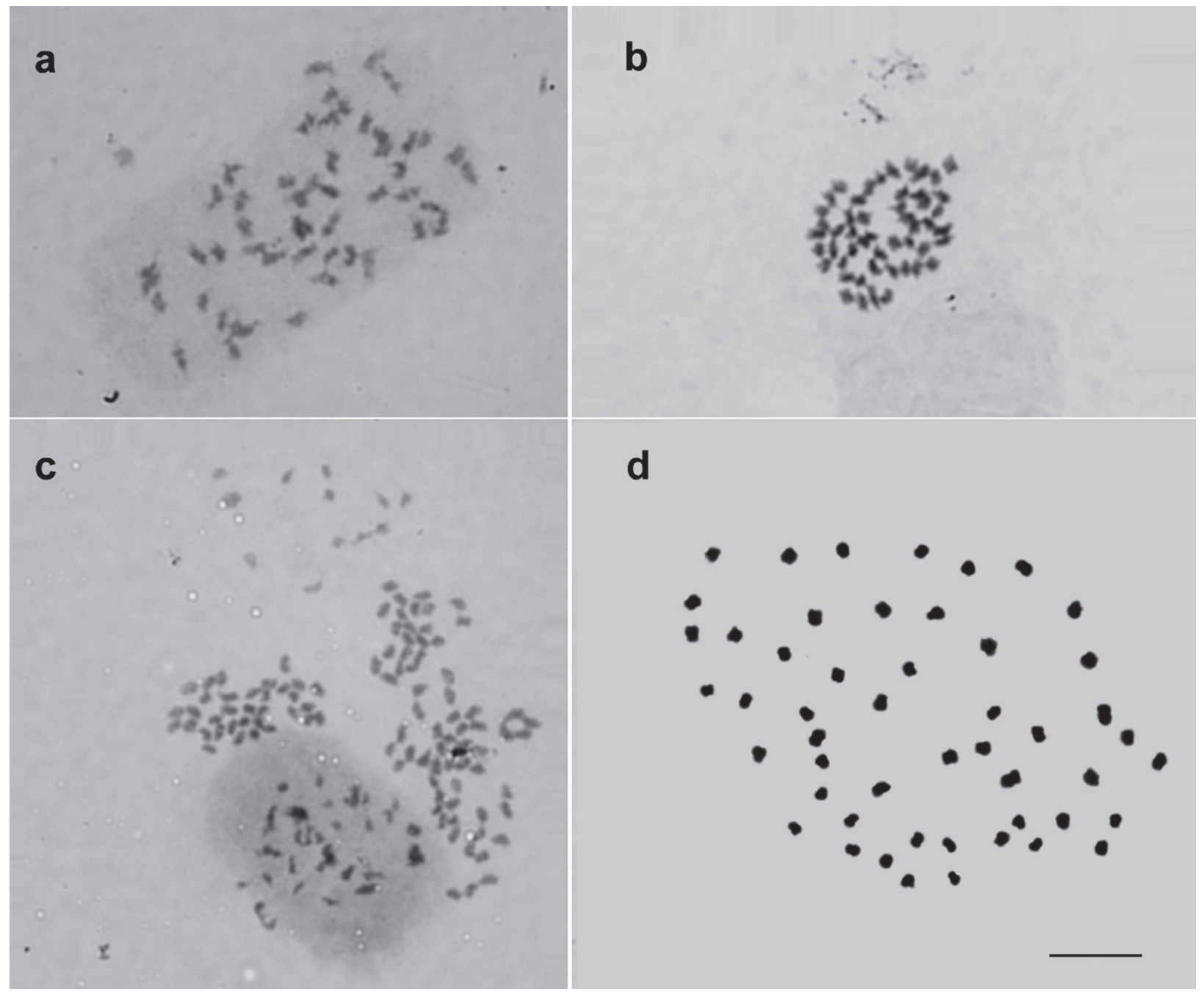

Figure 2 - Cytogenetic preparations of Pitcairnia flammea (L. B. Smith) L. B. Smith with chromosomes at different levels of chromatin compaction. (a) Late prophase with $2 \mathrm{n}=50$ chromosomes. (b) Early prometaphase exhibiting $2 \mathrm{n}=50$ chromosomes. (c) Overlapping interphase nuclei, prophase and prometaphase chromosomes. (d) Metaphase displaying $2 \mathrm{n}=50 \mathrm{chromosomes,} \mathrm{all}$ submetacentric. This adequate cytogenetic preparation showed well-spread chromosomes, with well-defined primary constriction, without chromatin damage and cytoplasmic background.

the Bromeliaceae family is not $\mathrm{x}=25$ chromosomes. Furthermore, the presence of an isolated chromosome (number 1) led to evidence of an allopolyploid origin for the genome of $P$. flammea.

Despite the advances made in cytogenetic studies, these analyses in Bromeliaceae have covered 10\% of all species of the group. This fact is due especially to the relatively high number, generally $2 n=50$, and the small total size of the chromosomes. Therefore, more cytogenetic studies in bromeliads have to be conducted aiming to expand the knowledge about their karyotype. For this end, karyotype researches must be allied to molecular cytogenetics, which can evidence the evolution of DNA sequences present in the chromosomes.

\section{FCM IN BROMELIACEAE}

FCM analyses have been used to estimate the nuclear DNA content and base composition (AT\% and $\mathrm{GC} \%$ ) of a few Bromeliaceae species 
(Favoreto et al. 2012). The determination of these values has contributed to studies on systematic and evolution (Ebert and Till 1997, Ramirez-Morillo and Brown 2001, Nunes et al. 2013), genetic diversity and reproductive biology (Sgorbati et al. 2004, Zonneveld et al. 2005, Favoreto et al. 2012) of bromeliads species.

Applied to Bromeliaceae species, FCM analyses have primarily aimed to determine their $2 \mathrm{C}$ nuclear DNA content in picograms (pg). Using this application, Arumuganathan and Earle (1991) reported, for the first time, the $2 \mathrm{C}$ nuclear value of two bromeliad species: Ananas bracteatus (Lindley) Schultes f. presented 2C $=0.920 \mathrm{pg}$, and A. comosus (Linnaeus) Merrill showed 2C $=1.090 \mathrm{pg}$.

Also applying FCM, Ebert and Till (1997) established the nuclear genome size of 47 species distributed in ten genera of the subfamily Pitcairnioideae. The values varied from $2 \mathrm{C}=0.600$ pg for Pitcairnia L'Heritier to $2 \mathrm{C}=1.860 \mathrm{pg}$ for Fosterella L. B. Smith (Table I).

Ramirez-Morillo and Brown (2001) measured the nuclear genome size of Cryptanthus species, with $2 \mathrm{n}=34$ or 36 chromosomes, and other bromeliads showing $2 \mathrm{n}=50$ chromosomes. The highest value of nuclear genome size was found for $C$. beuckeri $\mathrm{E}$. Morren $(2 \mathrm{C}=1.458 \mathrm{pg})$, and the lowest for C. schwackeanus Mez $(2 \mathrm{C}=0.710 \mathrm{pg})$. In relation to other species, Orthophytum saxicola (Ule) L. B. Smith showed the lowest DNA content, estimated at 2C $=0.640 \mathrm{pg}$ (Table I).

Sgorbati et al. (2004) used FCM to study the genetic diversity and reproductive biology of Puya raimondii Harms. These authors examined relationships between populations of this species and their reproductive mechanism for obtaining subsidies to delineate conservation strategies. Therefore, the $2 \mathrm{C}$ value of $P$. raimondii was estimated $(2 \mathrm{C}=1.130 \mathrm{pg})$, and the embryo was found to have a relative DNA content equivalent to $2 \mathrm{C}$ and $3 \mathrm{C}$ of the endosperm. This data revealed the sexual reproduction system in this species.
Zonneveld et al. (2005) measured the nuclear genome size of two bromeliad species, Tillandsia cyanea Linden ex K. Koch $(2 \mathrm{C}=2.200 \mathrm{pg})$ and Tillandsia usneoides (Linnaeus) Linnaeus ( $2 \mathrm{C}=2.500$ pg). In current analyses, Favoreto et al. (2012) reported the nuclear DNA content and base composition (AT\%) of 14 Bromeliaceae species, which ranged from $2 \mathrm{C}$ $=0.770 \mathrm{pg}$, for Billbergia horrida Regel, and $2 \mathrm{C}=$ $3.340 \mathrm{pg}$, for Tillandsia loliaceae Martius ex Schultes f. The base composition was AT $=60.26 \%$ for Vriesea racinae $\mathrm{L}$. B. Smith and $\mathrm{AT}=66.05 \%$ for Billbergia tweedieana Baker (Table I).

Nunes et al. (2013) reported the $2 \mathrm{C}$ value $(1.440 \mathrm{pg})$ and base composition (AT $=64.28 \%$ and $\mathrm{GC}=35.72 \%)$ of $P$. flammea . According to these authors, the nuclear DNA content of $P$. flammea can be considered relatively small compared to the nuclear $2 \mathrm{C}$ value of most angiosperms. As stated by Bennett and Leitch (2011), the nuclear DNA content of angiosperms ranges from an equivalent minimum value of $2 \mathrm{C}=0.1296 \mathrm{pg}$ for Genlisea margaretae Hutch to a maximum value of $2 \mathrm{C}=304.46 \mathrm{pg}$ for Paris japonica (Franch. \& Sav.) Franch.

Different studies have reported the nuclear DNA content for various Bromeliaceae species (Table I). Similarly to P. flammea (Nunes et al. 2013), these nuclear $2 \mathrm{C}$ values are considered relatively small, characterizing a constant in the bromeliad group. Thus, regarding a possible reconstruction of the ancestral nuclear genome $(2 \mathrm{C}=3.700 \mathrm{pg})$ for the monocot group, a significant decrease in the DNA content of bromeliads can be observed throughout their genomic evolution.

Despite concerted efforts towards the $2 \mathrm{C}$ value estimation for the Bromeliaceae family, FCM analyses are scarce, and only $2.3 \%$ of bromeliads have had their genome size established (Table I).

\section{PERSPECTIVES AND CONCLUSION}

Cytogenetic studies in Bromeliaceae have evolved with the establishment of a concise number of $2 n=$ 50 chromosomes for most of the analyzed species. 
Additionally, karyological analysis of $P$. flammea allowed initial inferences on the karyotype evolution of bromeliads. Thus, as a starting point for further research, it should be assumed that $\mathrm{x}=25$ might not be the basic Bromeliaceae chromosome number, a fact that could be the subject of new cytogenetic studies.

Bromeliaceae FCM approaches are incipient, since only few species have already had their $2 \mathrm{C}$ nuclear DNA content estimated this way. FCM provides fast and reliable analyses, playing an important role in genetic diversity and systematic studies and in assisting cytogenetic research, by enabling immediate DNA ploidy level determination. Considering these facts, not to expand FCM studies means to delay the gain of Bromeliaceae genome knowledge. Therefore, more different bromeliads species ought to have their $2 \mathrm{C}$ value determined, with the aim of supporting karyotype studies.

In conclusion, in order to reach a robust result regarding the process of bromeliad karyotype evolution, it is time to initiate a combination of classical and molecular cytogenetics of Bromeliaceae species, allied with a greater number of FCM analyses.

\section{ACKNOWLEDGMENTS}

The authors are grateful to Conselho Nacional de Desenvolvimento Científico e Tecnológico (CNPq, Brasília, DF, Brazil), Fundação de Amparo à Pesquisa e Inovação do Espírito Santo (FAPES, Vitória, ES, Brazil), and Coordenação de Aperfeiçoamento de Pessoal de Nível Superior (CAPES, Brasília, DF, Brazil) for financial support.

\section{RESUMO}

Análises citogenéticas e de citometria de fluxo em Bromeliaceae têm sido realizadas para esclarecer aspectos de sistemática e evolução. $\mathrm{O}$ cariótipo das bromeliáceas apresenta cromossomos em número relativamente elevado, morfologicamente similares e com tamanho pequeno. Esses aspectos têm dificultado a determinação correta do número e da caracterização de cromossomos. Os autores estabeleceram um número básico de cromossomos de $\mathrm{x}=25$ para Bromeliaceae. Recentemente, uma análise cariomorfológica revelou que $\mathrm{x}=25$, já não é o número básico de cromossomos de Bromeliaceae, cujo genoma pode ter origem poliploide. Além da caracterização citogenética, o conteúdo $2 \mathrm{C}$ de DNA de Bromeliaceae tem sido mensurado. O conteúdo de DNA nuclear tem variado de $2 \mathrm{C}=0,60$ a $2 \mathrm{C} 3,34$ picogramas. Portanto, em comparação com a maioria das angiospermas, o conteúdo $2 \mathrm{C}$ de DNA das espécies de Bromeliaceae e o tamanho de seus cromossomos podem ser considerados relativamente pequenos. Apesar de alguns avanços, os dados citogenéticos e de citometria de fluxo são extremamente escassos nesse táxon. Nesse contexto, a presente revisão reporta o estado da arte no que se refere à caracterização do cariótipo e o mensuramento do conteúdo de DNA nuclear em Bromeliaceae, enfatizando os principais problemas e sugerindo soluções potenciais e ideias para pesquisas futuras.

Palavras-chave: alopoliploide, bromélias, citogenética, citometria de fluxo, evolução do genoma.

\section{REFERENCES}

APG III. 2009. An update of the Angiosperm Phylogeny Group classification for the orders and families of flowering plants: APG III. Bot J Linn Soc 161: 105-121.

ARUMUGanAthan K AND EARLE ED. 1991. Nuclear DNA content of some important plant species. Plant Mol Biol Rep 9: 208-218.

Bellintani MC, Cotias-DE-Oliveira ALP AND Assis JGA. 2005. Chromosomal Evolution of Bromeliaceae. Cytologia 70: 129-133.

BENNETT MD AND LeITCH IJ. 2011. Nuclear DNA amounts in angiosperms: targets, trends and tomorrow. Ann BotLondon 107: 467-590.

BILlings FH. 1904. A study of Tillandsia usneoides. Bot Gaz 38: 99-121.

Brown GK AND GILMARTIN AJ. 1989. Chromosome numbers in Bromeliaceae. Am J Bot 76: 657-665.

Carvalho CR, Clarindo Wr, PraÇa MM, AraúJo FS AND CARELS N. 2008. Genome size, base composition and karyotype of Jatropha curcas L., an important biofuel plant. Plant Sci 174: 613-617.

Ceita GO, Assis JGA, Guedes MLS and Cotias-DeOLIVEIRA ALPC. 2008. Cytogenetics of Brazilian species of Bromeliaceae. Bot J Linn Soc 158: 189-193.

Cotias-DE-Oliveira ALP, ASSIS JGA, BELlintani MC, ANDRADE JCS AND GUEDES MLS. 2000. Chromosome numbers in Bromeliaceae. Genet Mol Biol 23: 173-177. 
Cotias-De-Oliveira ALP, Assis JGA, Ceita O, PAlmeira ACL AND GUEDES MLS. 2004. Chromosome number for Bromeliaceae species occurring in Brazil. Cytologia 69: 161-166.

EBERT I AND TILL W. 1997. Nuclear genome size in Pitcairnioideae (Bromeliaceae) with emphasis on the genus Pitcairnia. Abstracts, Angiosperm Genome Size Discussion Meeting., p. 15. Royal Botanical Gardens, Kew. 11-12 September 1997.

FAVORETO FC, CARVALHO CR, LIMA ABP, FERREIRA A AND ClARINDO WR. 2012. Genome size and base composition of Bromeliaceae species assessed by flow cytometry. Plant Syst Evol 298: 1185-1193.

Gitaí J, Horres R AND BENKO-ISEPPON AM. 2005. Chromosomal features and evolution of Bromeliaceae. Plant Syst Evol 253: 65-80.

GIVNISH TJ ET AL. 2011. Phylogeny, adaptive radiation, and historical biogeography in Bromeliaceae: insights from an eight-locus plastid phylogeny. Am J Bot 98: 872-895.

LINDSCHAU M. 1933. Beiträge zur Zytologic der Bromeliaceae. Planta 20: 506-530.

LOUZADA RB, PALMA-SILVA C, CORRÊA AM, KaLTCHUK-SANTOS E AND WANDERLEY MGL. 2010. Chromosome number of Orthophytum species (Bromeliaceae). Kew Bull 65: 53-58.

MARCHANT CJ. 1967. Chromosome evolution in the Bromeliaceae. Kew Bull 21: 161-168.

MCWilliams E. 1974. Chromosome number and evolution. In: Smith LB and Downs RJ (Eds), Bromeliaceae (Pitcairnioideae). Flora Neotropica Monographs. Hafner Press, New York, 14: 33-40.
Nunes ACP, Nogueira EU, Gontijo ABPL, CARvalho CR AND CLARINDO WR. 2013. The first karyogram of a Bromeliaceae species: an allopolyploid genome. Plant Syst Evol 299: 1-8.

Palma-Silva C, SAntos DG, KaltchuK-Santos E AND BodANESE-ZANETTINI MH. 2004. Chromosome numbers, meiotic behavior and pollen viability of species of Vriesea and Aechmea genera (Bromeliaceae) native to Rio Grande do Sul, Brazil. Am J Bot 91: 804-807.

RAMÍREZ-MORILLO IMAND BROWN GK. 2001. The origin of the low chromosome number in Cryptanthus (Bromeliaceae). Syst Bot 26: 722-726.

SGORBATI S ET AL. 2004. Survey of genetic diversity and reproductive biology of Puya raimondii (Bromeliaceae), the endangered queen of the Andes. Plant Biology 6: 222-230.

SHARMA AK AND GHOSH I. 1971. Cytotaxonomy of the family Bromeliaceae. Cytologia 36: 237-247.

VARADARAJAN GS AND BROWN GK. 1985. Chromosome number reports LXXXIX. Taxon 34: 727-730.

VersieuX LM. 2009. Sistemática, Filogenia e Morfologia de Alcantarea (Bromeliaceae). Tese (Doutorado em Botânica). Instituto de Biociências. Universidade de São Paulo, 2009.

WEISS HE. 1965. Étude caryologique et cyto-taxonomique de quelques Broméliaceés. Mémories du Muséum National d'Histoire Naturelle 16: 9-38.

ZonNeVEld BJM, Leitch IJ AND BenNeT D. 2005. First nuclear DNA amounts in more than 300 Angiosperms. Ann Bot-London 96: 229-244. 
